\title{
Advanced Information Technologies in Future Computing Environments
}

\author{
Jongsung Kim • Hamid R. Arabnia • Jiqiang Lu
}

Published online: 18 June 2013

C) Springer Science+Business Media New York 2013

Information Technology (IT) would stand for all of continuously evolving and converging technologies, including digital convergence, multimedia convergence, intelligent applications, embedded systems, mobile and wireless communications, bio-inspired computing, grid and cloud computing, semantic web, user experience and HCI, security and trust computing and so on, for satisfying our ever-changing needs. In past three decades, IT influenced and changed every aspect of our lives and our cultures. Without various IT-based applications, it would be difficult to process information efficiently, to communicate information conveniently, and to keep information stored securely. In future computing environments (cloud and cluster computing, pervasive and ubiquitous computing, etc.), IT will play a very important role in convergence of computing, communication and all other computational sciences and applications. IT will also influence the future world's various areas, including science, engineering, industry, business, law, politics, culture, medicine, and so on.

This special issue is to foster the dissemination of state-of-the-art research in all advanced information technologies in future computing environments, including their models, services, and novel applications associated with their utilization. This special issue is aimed to solicit research results on the following topics of interest, but not limited:

- Hybrid Information Technology

- High Performance Computing

\footnotetext{
J. $\operatorname{Kim}(\varangle)$

Department of Mathematics, Kookmin University, 77, Jeongneung-Ro, Seongbuk-Gu, Seoul 136-702, Republic of Korea

e-mail: jongsung.k@gmail.com

H. R. Arabnia

Department of Computer Science, University of Georgia, 415 Boyd Building, Athens, GA 30602-7404, USA

e-mail: hra@cs.uga.edu

J. Lu

Institute for Infocomm Research, 1 Fusionopolis Way, \#19-01 Connexis, South Tower, Singapore 138632, Singapore

e-mail: lvjiqiang@hotmail.com
} 
- Cloud and Cluster Computing

- Ubiquitous Networks and Wireless Communications

- Digital Convergence

- Multimedia Convergence

- Intelligent and Pervasive Applications

- Security and Trust Computing

- IT Management and Service

- Bioinformatics and Bio-Inspired Computing

- Database and Data Mining

- Knowledge System and Intelligent Agent

- Game and Graphics

- Human-centric Computing and Social Networks

We received many manuscripts. Each manuscript was blindly reviewed by at least three reviewers consisting of guest editors and external reviewers. After the review process, seventeen manuscripts were finally selected for this Special Issue. The selected manuscrypts are as follows:

1. Rule-based Security Capabilities Matching for Web Services, Bo Yu, Lin Yang, Yongjun Wang, Bofeng Zhang, Yuan Cao, Linru Ma, Xiangyang Luo

2. Time-Efficient Handover Using Enhanced Route Optimization in Global PMIPv6, Dongxu Jin, Fei Shi, JoonSup Chin, JooSeok Song

3. A Semantic Approach for Transforming XML Data into RDF Ontology, Pham Thi Thu Thuy, Young-Koo Lee, Sungyoung Lee

4. An Analysis of Reducing Communication Delay in Network-on-Chip Interconnect Architecture, Hasan Furhad, Mohammad Haque, Cheol-Hong Kim, Jong-Myon Kim

5. Repackaging Attack on Android Banking Applications and Its Countermeasures, JinHyuk Jung, Ju Young Kim, Hyong-Chan Lee, Jeong Hyun Yi

6. Effective and Comfortable Power Control Model Using Kalman Filter for Building Energy Management, Safdar Ali, Do-Hyeun Kim

7. ODSA: Chord-based Object Discovery Service Architecture for the Internet of Things, De-Gang Xu, Lei-Hua Qin, Jong Hyuk Park, Jing-Li Zhou

8. Low-cost Web Service Discovery based on Distributed Decision Tree in P2P Environments, Shangguang Wang, Xilu Zhu, Qibo Sun, Hua Zou, Fangchun Yang

9. ELACCA: Efficient Learning Automata Based Cell Clustering Algorithm for Wireless Sensor Networks, Neeraj Kumar, Jongsung Kim

10. VRAS: A Lightweight Local Resource Allocation System for Virtual Machine Monitor, Hai Jin, Wei Gao, Song Wu, Xuanhua Shi

11. Contents Recommendation Method Using Social Network Analysis, Jong-Soo Sohn, Un-Bong Bae, In-Jeong Chung

12. A Novel Security Scheme for the Smart Grid and SCADA Networks, Hussam Al Hamadi, Chan Yeun, Mohamad Zemerly

13. Technology Acceptance Model for the Use of Tablet PCs, Eunil Park, Angel P. del Pobil

14. An Adaptable Job Submission System based on Moderate Price-Adjusting Policy in Market-based Grids, Asgarali Bouyer, Bahman Arasteh

15. A Study on Project Selection Framework for Future ICT Technologies, Yanghoon Kim, Hangbae Chang

16. User Requirement Analysis and IT Framework Design for Smart Airports, Sei-Chang Sohn, Kee-Woong Kim,Chulung Lee 


\section{An Efficient Multi-Path Self-Organizing Strategy in Internet of Things, Tie Qiu, Weifeng Sun, Yuanchao Bai, Yu Zhou}

Finally, we would like to thank all authors for their contributions to this special issue. We also extend our thanks to the following external reviewers for their excellent job in reviewing the manuscripts: Seungmin Rho, Sangho Park, Sang-Soo Yeo, Han Xu, Jun Wu, Claudio Ardagna, Jen Wei Hsieh, Jenq Muh Hsu, Zia Tanveer, Peter Varman, Qishi Wu, Soshi Masakazu, Zhenglu Yang, ChihLin Hu, ChunYang Ye, Shen Haifeng, KueiPing Shih, Pfisterer Dennis, Ce Kuen Shieh, Shu Ching Chen, Wei Huang, Yo Ping Huang, Ruay Shiung Chang, Taeshik Shon, Yang Sun Lee, Changhoon Lee, Simone Ludwig, Dragan Ivetic, Sanjukta Bhowmick, Ayesha Naureen.

\section{Author Biographies}

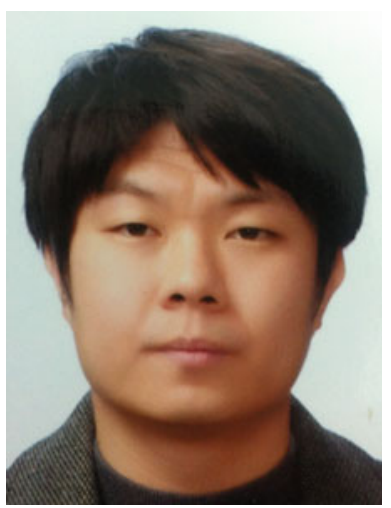

Jongsung Kim received his Bachelor and Master degrees in Mathematics from Korea university, Korea in 2000 and 2002, respectively. He received double Doctoral degrees completed in November 2006 and February 2007 at the ESAT/COSIC group Katholieke Universiteit Leuven and at Engineering in Information Security of Korea University, respectively. He had been a Research Professor of Center for Information Security Technologies (CIST) at Korea University, Korea, from March 2007 till August 2009, and an assistant professor of department of e-business at Kyungnam University, Korea, from September 2009 till February 2013. Dr. Kim has been an assistant professor of department of mathematics at Kookmin University, Korea, since March 2013. Dr. Kim has published about 60 research papers in international journals and conferences. He has been serving as chairs, program committee, or organizing committee chair for many international conferences and workshops. He is editorial board member of International Journal of Information Technology, Communications and Convergence (IJITCC), International Journal of Communication Networks and Distributed Systems (IJCNDS), InderScience and Journal of Convergence (JoC), FTRA Publishing, and Human-centric Computing and Information Sciences (HCIS), Springer. In addition, he has been serving as a Guest Editor for international journals by some publishers: Springer, Elsevier, John Wiley, Oxford Univ. press, Inderscience. His research interests include security issues, cryptography, ubiquitous computing systems and digital forensics.

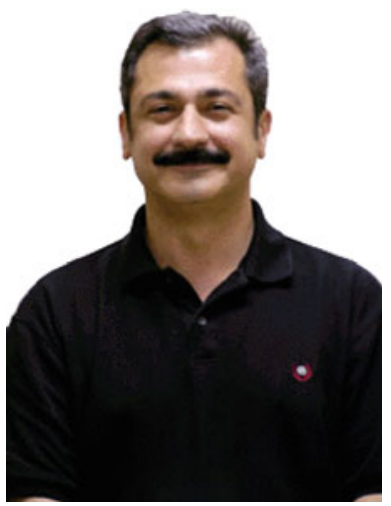

Hamid R. Arabnia is an author, editor of research books, educator, and researcher. He received a $\mathrm{Ph} . \mathrm{D}$. degree in Computer Science from the University of Kent (Canterbury, England) in 1987. In 1987, he worked as a Consultant for Caplin Cybernetics Corporation (London, England), where he helped in the design of a number of image processing algorithms that were targeted at a particular parallel machine architecture. Arabnia is currently a Full Professor of Computer Science at University of Georgia (Georgia, USA), where he has been since October 1987. His research interests include Parallel and distributed processing techniques and algorithms, interconnection networks, and applications (in particular, in image processing and other computational intensive problems). Dr. Arabnia has chaired many national and international conferences and technical sessions in these areas; he is the chair of WORLDCOMP annual congress. He is Editor-in-Chief of the Journal of Supercomputing (which is one of the oldest journals published by Springer) and is on the editorial and advisory boards of 26 other journals and magazines. Dr. Arabnia is the recipient of William F. Rockwell, Jr. Medal for promotion of multi-disciplinary research (Rockwell Medal is International Technology Institute's highest honor). In 2000, Dr. Arabnia was inducted to the World Level of the Hall of Fame for Engineering, Science and Technology (The World Level is the highest possible level for a living person). He has received a number of awards, including, The Johns Hopkins University National Search (Certificate of Achievement) in recognition of his 
contributions to the national program for enhancing the quality of life for people with disabilities through the application of computing technology (presented to him in December 1991-signatories: co-directors of the National Search and President of Johns Hopkins U.) In 2006, Dr. Arabnia received the Distinguished Service Award in recognition and appreciation of his contributions to the profession of computer science and his assistance and support to students and scholars from all over the world; this award was formally presented to him on June 26, 2006 by Professor Barry Vercoe (Massachusetts Institute of Technology / MIT). More recently (October 14, 2007), he received an "Outstanding Achievement Award in Recognition of His Leadership and Outstanding Research Contributions to the Field of Supercomputing". This award was formally presented to him at Harvard University Medical School (signatories: Lawrence O. Hall, President of IEEE/SMC; Zhi-Pei Liang, Vice President of IEEE/EMB; Jack. Y. Yang, General Chair of IEEE BIBE and Harvard University; Mary Qu Yang, Chair of Steering Committee, IEEE BIBE and NIH).Dr. Arabnia has published extensively in journals and refereed conference proceedings as well as edited numerous books. He has been a Co-PI on $\$ 7,139,525$ externally funded projects/initiatives (mainly via Yamacraw-includes some UGA matching) and on $\$ 103,453$ internally funded projects (as of January 2009). He has also contributed projects for justification for equipment purchase (grant proposals worth over \$3 Million-awarded). During his tenure as Graduate Coordinator of Computer Science (August 2002-January 2009), he secured the largest level of funding in the history of the department for supporting the research and education of graduate students (Ph.D., M.S.). Dr. Arabnia has delivered numerous number of keynote lectures at international conferences; most recently at (since September 2008): The 14th IEEE International Conference on Parallel and Distributed Systems (ICPADS'08, Australia; http://www.deakin.edu.au/conferences/icpads2008/speakers.php); International Conference on Future Generation Communication and Networking (FGCN 2008 / IEEE CS, Sanya/China; http://www.sersc.org/FGCN2008/speakers.php); The 10th IEEE International Conference on High Performance Computing and Communications (HPCC-08, Dalian/China), ... He has also delivered a number of "distinguished lectures" at various universities.

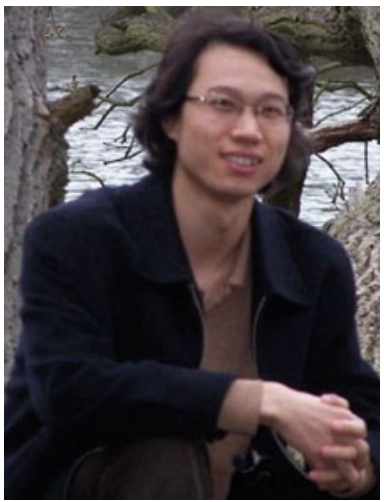

Jiqiang Lu was born in Gaomi city, Shandong Province, P.R. China. He received a B.Sc. degree in Applied Mathematics from Yantai University in 2000, a M.Eng. degree in Information and Communication Engineering from Xidian University in 2003, and a Ph.D. degree from the University of London in 2008. He was with Department of Science and Technology of Shandong Province, Information and Communication University, ONETS Wireless and Internet Security Tech. Co. Ltd., Huawei Technologies, Co. Ltd., Eindhoven University of Technology, and École Normale Supérieure. He is now a scientist in Institute for Infocomm Research, Agency for Science, Technology and Research. 